\title{
Sensors for geometric accurate measurements in manufacturing
}

\author{
Albert Weckenmann ${ }^{1}$, Stephanus Büttgenbach ${ }^{2}$, Özgür Tan ${ }^{1}$, Jörg Hoffmann ${ }^{1}$, Alexander Schuler ${ }^{1}$ \\ ${ }^{1}$ Chair Quality Management and Manufacturing Metrology (QFM); University Erlangen; \\ albert.weckenmann@qfm.uni-erlangen.de \\ ${ }^{2}$ Institute for Microtechnology; University of Technology Brunswick \\ s.buettgenbach@tu-bs.de
}

Main idea for every dimensional measurement is the detection of the surfaces or a group of points on an object by means of a probing system. The application of this fundamental idea in the field of micro and nano metrology, with size aspects or part tolerances in the sub micrometer regions, is very challenging. This task requires the application of precise length measuring systems for setting up a coordinate system and suitable sensors. The aim of this paper is to demonstrate recent scientific and technological developments by means of the most promising existing applications in this field.

\section{INTRODUCTION}

Due to the downscaling of components for miniaturized systems, simultaneous advancements of measurement systems are necessary. Miniature components like e.g. micro gears or MEMS feature size aspects or three dimensional structures in the dimension of micrometers with often sub micrometer tolerances. Keeping in mind that according to Bernt's "golden rule" of metrology the measurement uncertainty should be about $10 \%$ of the tolerance to be checked, resolution in the nanometer order and measurement uncertainty of a few ten nanometers would be appropriate. Regular coordinate measurement machines with e.g. tactile probes can't meet these requirements or are not able to probe complex structures at all. Therefore several promising concepts concerning CMMs and sensors have been developed and tested worldwide.

\section{BASIC COMPONENTS OF PRECISION CMMS}

Additional to developments in sensor technology, there has been made a great effort to obtain coordinate measurement machines with higher resolution up to sub-nanometer. Several approaches have been applied, which result in different resolutions or working ranges. According to the latest activities, high precision coordinate measuring machines can be subdivided into three main categories; metrological scanning probe microscopes MSPM, nanometer resolution coordinate measuring machines NCMM and micro coordinate measuring machines MCMM. In order to get an overview of these instruments and the parts which especially account for the improvements, it is important to look into the basic components of these instruments.

\subsection{METROLOGY FRAME AND AXIS DESIGN}

To achieve a measurement system with nanometer resolution, a design which is resistant to thermal influences and mechanical noise is necessary. Due to the fact that vibration and thermal drift easily reach amplitudes of sub-micrometers, these factors should be avoided or compensated.

A first step in this direction is the separation of the motion system and the metrology frame, which are both located on the machine base. Like in common CMMs, the base is usually made of granite and serves not only as temperature stable base but also as a passive vibration dampener. In some instruments, like Werth Videocheck UA, additional dampening systems are already part of the CMM [1]. The mechanical and thermal isolation of the metrology frame from the motion system serves the load and strain avoidance as well as the reduction of vibration and thermal influences resulting from the drives. This is important as the frame carries the length measuring system for the axes and the probing system. To further reduce the influence of thermal effects, materials like Zerodur or Invar with a low thermal coefficient are used for the metrology frames.

Concerning the length measuring system, the application of laser interferometers is a common approach, allowing a special Abbé-arrangement to eliminate first order Abbé errors. As seen in figure 1, the beams from the interferometers intersect in one single point which is the probing point of the installed sensor. This requires that the sensor is fixed and only the workpieces move to keep this arrangement, 
alternatively the axes would have to follow the sensor movement. CMMs based on the first concept are e.g. the SIOS NMM-1 and the IBS Isara [2], [3].

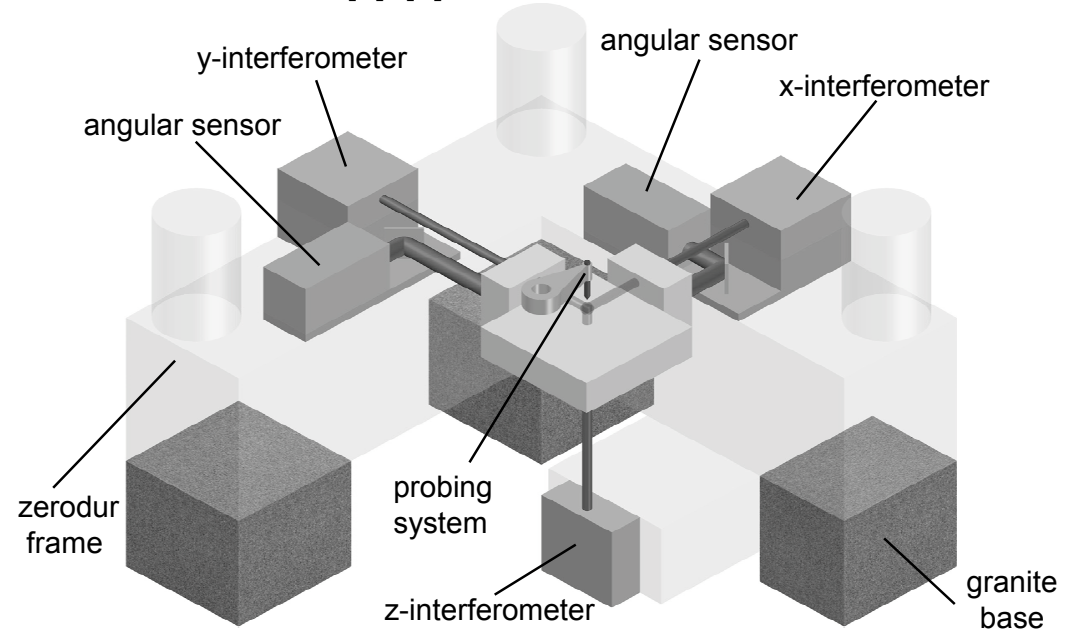

figure 1: NMM-1: metrology frame and axes arrangement, (figure according to SIOS documentation)

Further concepts using other length measuring systems would be glass scales for larger working ranges and high resolution capacitive systems for small working ranges.

\subsection{Guiding and motion systems}

Concerning the implementation of the guiding systems in high precision CMMs, different approaches are possible. In the first approach, air bearings, which are commonly used in regular scale coordinate metrology, have been adopted to CMMs. This approach has been realised e.g. by Ruijl [3] and applied in the Ultra Precision CMM, now available as the IBS Isara, having the advantage of being frictionless which results in a decrease of required driving power. Air bearings show a certain clearance depending on the actual air pressure and load. Furthermore they emit exhaust air which might affect the machine performance.

Another approach is to use flexure hinges and levers to compose transversal movements, like in the kinematic system of the PTB Veritekt [4], figure 2. High reproducibility and low friction are the advantages of these guiding systems. In comparison to the large size of this kinematics they have a low movement range and they show non-linear behaviour. Several new concepts in the design of flexure hinges are being developed trying to compensate the problems.

A third approach, which has been realised at the SIOS NMM-1 [2], is based on the application of high precision roller bearings in combination with an advanced non-linear position and motion control system.
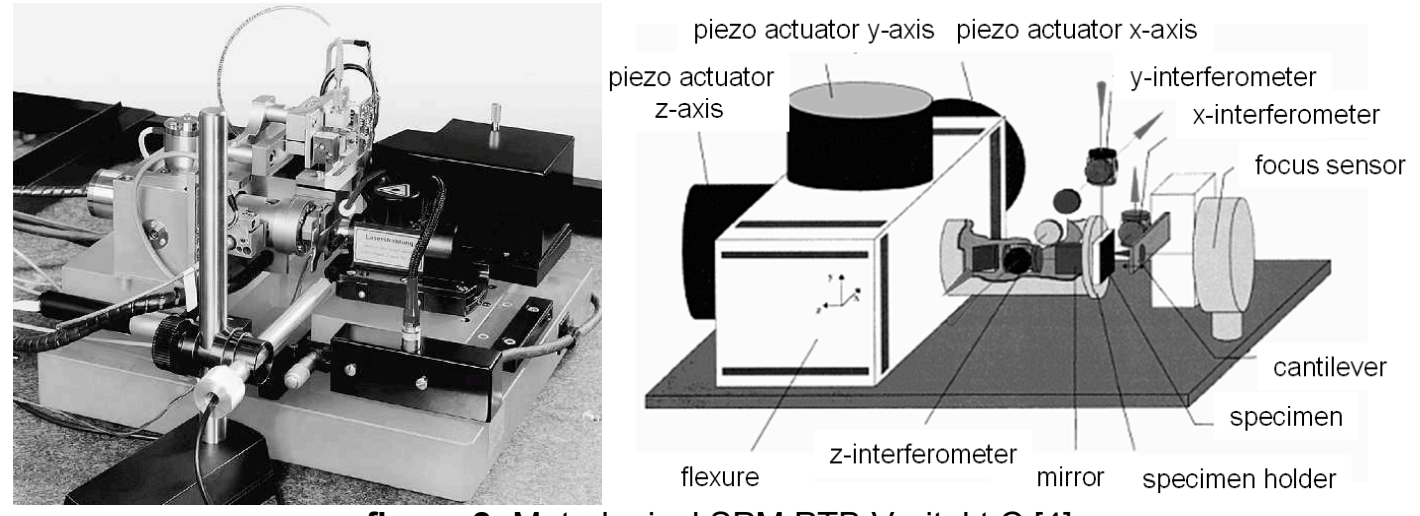

figure 2: Metrological SPM PTB Veritekt C [4]

There are also different possibilities for the driving system depending on the required movement range and acceleration. For very small working ranges $(<100 \mu \mathrm{m})$ mainly monolithic and stacked piezo actuators are used. High resolution capability down to sub-nanometer level, high acceleration and low power consumption are their advantages. Due to the adverse ratio of movement to total size it is not possible to span large working ranges with a single compact piezo and furthermore a closed loop control 
is necessary because of large non-linearity and hysteresis effects. Possible solutions for the low working range are already available in the form of repeated stepwise movement e.g. with friction based assemblies [5]. Apart from piezo systems one can usually find Lorentz actuators for larger working ranges like e.g. in NMM-1.

\subsection{COMMERCIAL INSTRUMENTS}

\subsubsection{Metrological scanning probe microscopes MSPM}

MSPMs are characterized by a very small measuring volume of typically $<100 \mu \mathrm{m}$ in $\mathrm{x}$ and $\mathrm{y}$ axis and 10 $20 \mu \mathrm{m}$ in z-direction. Resolution reaches usually sub nanometer level. Example for this category of instruments is the Veritekt C of the German NMI PTB in Braunschweig (figure 2). It is based on the commercial Zeiss AFM Veritekt (not available any more), which is improved with three SIOS laser interferometers in order to maintain the regulations of positioning.

\subsubsection{Nanometer resolution coordinates measuring machines NCMM}

For the second category there are already two commercial products available, the IBS ISARA (Netherlands) and the SIOS NMM-1 (Germany), figure 3. Their basic setup is shown in figure 1. The SIOS machine has a measuring range of $25 \times 25 \times 5 \mathrm{~mm}^{3}$ and a resolution of $0.1 \mathrm{~nm}$ [6], the volumetric positioning uncertainty is claimed to be about $10 \mathrm{~nm}$. The ISARA has a range of $100 \times 100 \times 40 \mathrm{~mm}^{3}$ and a resolution of $1.6 \mathrm{~nm}$. Claimed volumetric positioning uncertainty is $30 \mathrm{~nm}$ [3].
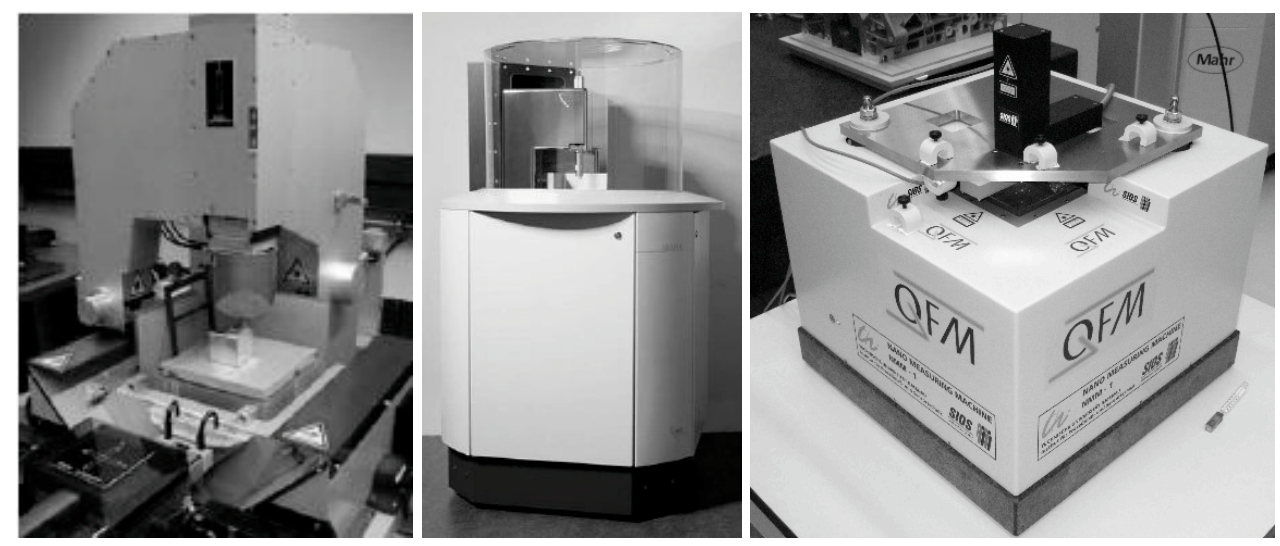

figure 3: Nano CMMs IBS ISARA (left, source: METAS, IBS) and SIOS NMM

\subsubsection{Micro coordinate measuring machines MCMM}

The first commercial micro CMM is the Zeiss F25 based on a design by Vermeulen (figure 4, [7]). Its setup is quite different compared to all other introduced systems. The scales of that machine are mounted onto intermediate bodies between machine base and ram and are moving together with the probing system in order to minimize Abbé-errors. This machine is specified with a volumetric measurement uncertainty of $250 \mathrm{~nm}$ in a volume of $100 \times 100 \times 100 \mathrm{~mm}^{3}$.
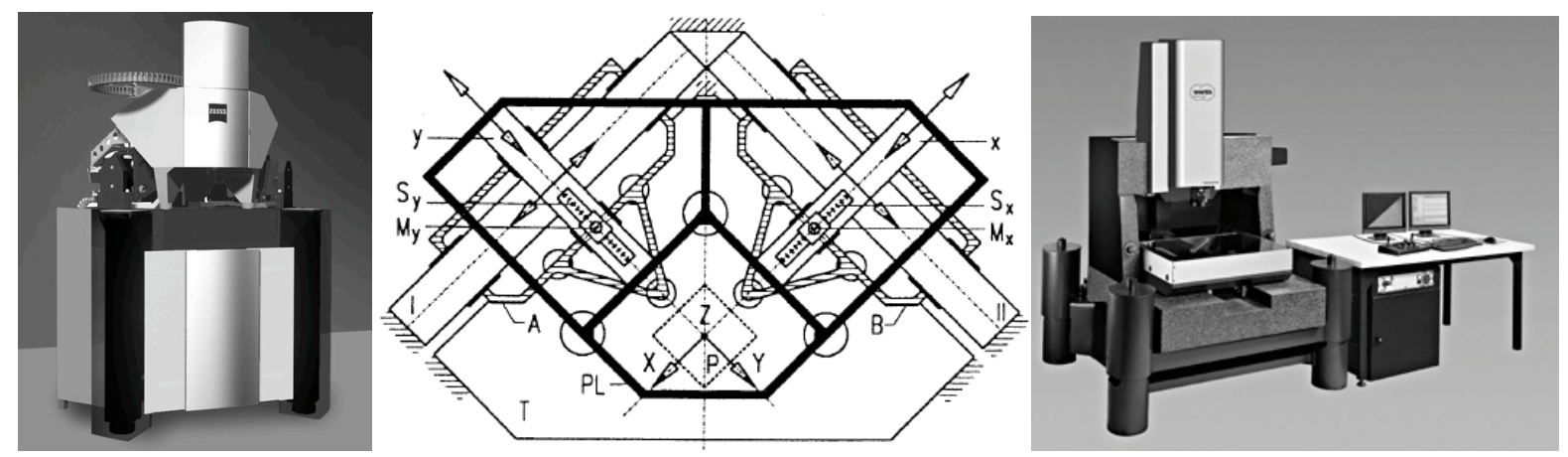

figure 4: Commercial micro CMM Zeiss F25 and its principal set-up [7], Werth Videocheck UA 400 [8] 
A second commercial micro CMM would be the Werth Videocheck UA 400 with a large working range of $400 \times 400 \times 200 \mathrm{~mm}^{3}$ and a length measurement error below $0.3 \mu \mathrm{m}$ [8]. It is designed as a multisensor CMM and uses e.g. the Werth Fiber Probe.

\section{Sensors}

In order to get a high precision measurement system, a suitable probing system for the CMM is needed to use the full capabilities of the high resolution axes. A wide variety of sensors with their own advantages and disadvantages are available [9],[10].

\subsection{Tactile probes}

The first and very important group of these sensors are tactile probes. This established and well known technique suffers from certain problems when micro structures have to be probed and nanometer resolution is needed. Along with the needed reduction of the probing sphere diameter like e.g. $0.3 \mathrm{~mm}$ goes a disproportionate rise in hertzian pressure between the sphere and the workpiece. This leads to surface damage of the workpiece and has to be compensated by reducing the probing force. Furthermore a lighter sphere and stylus shaft are used to reduce the force component by mass inertia. Therefore current developments focus on achieving miniaturized probing systems with a sensitivity of milli- or even micronewton. Some promising concepts combine the probing stylus via a flexible bar structure or membrane to piezoresistive or capacitive sensors to raise their sensitivity. Examples would be the capacitive microprobe developed at the National Physics Laboratory in the UK [11], now available at IBS Precision Engineering, or the piezoresistive probe developed at the Technical University of Braunschweig and the PTB [12]. The latter one is a monolithic design made from etched silicon. The stylus resides on a boss at the center of an etched membrane with diffused piezoresistive elements. By etching different membrane shapes and bar structures, the stress induced by the probing force can be better directed to increase the transducer sensitivity. Recent developments use these probes in an array for parallel probing to reduce measurement time [13], [14].

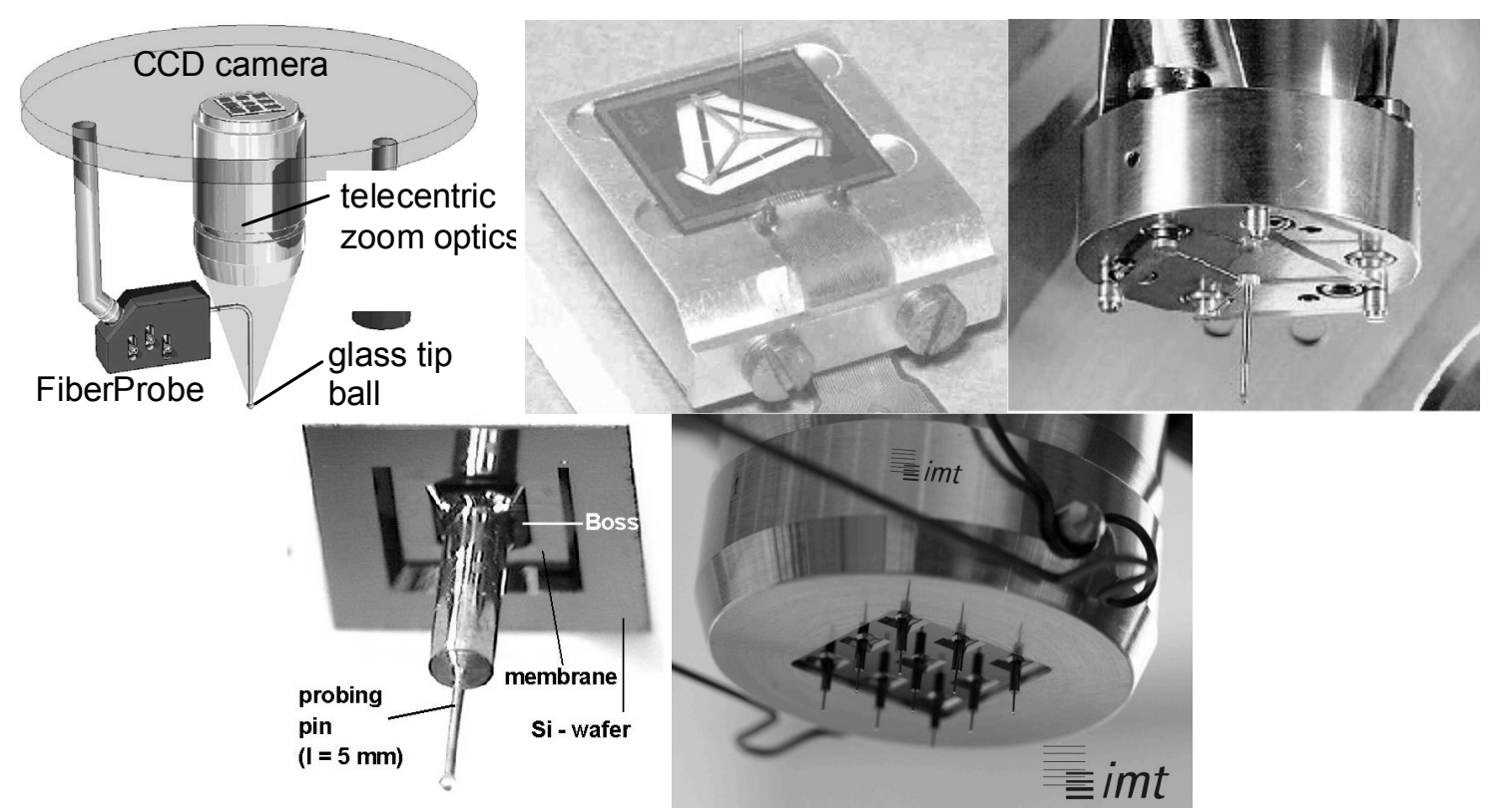

figure 5: the first commercial micro probing systems (top row from left to right: Werth FiberProbe; TU/E microprobe, source Schellekens; NPL microprobe, source NPL; bottom row microprobe and probe array, source PTB and TU Braunschweig)

Usually the microprobes are designed to detect not only the probing force but also its direction to realise a 3D probing system. Depending on the layout of the sensors a displacement of the stylus can be directly or indirectly derived from the sensor readings. For example the 3D touch probe developed at METAS with inductive transducers separates the force components via flexure hinges and shows the same probing force in all directions [15]. The microprobe from the TU Braunschweig also separately detects the force components by corresponding wiring of the wheatstone measurement bridges.

Other concepts to achieve a tactile microprobe use novel working principles to detect the probing sphere movement. For example Mitutoyo induces vibration to a stylus and detects changes in the amplitude, 
when the probing sphere gets in contact [16]. Another principle, which was developed by the PTB, is the optical detection of an illuminated glass sphere [17], also known as the Werth Fiber Probe.

Commercially available probing systems for micro coordinate measuring machines are up to now the 2D and 3D Werth FiberProbe, the 3D microprobes developed by Technical University of Eindhoven TU/E and the National Physics Laboratory of the UK NPL, as well as the 3D silicon microprobe developed by PTB and Technical University of Braunschweig, figure 5.

\subsection{Optical sensors}

Apart from tactile sensors there exist also several optical concepts. Some examples of them would be the chromatic sensor (figure 6) or the focus sensor. The measurement principle of the chromatic sensor is based on the chromatic aberration of white light. As the focus length of the lens depends on the refractive index, a lens with a strong chromatic aberration will show the focus point of the different wavelengths at different positions along the optical axis. The height information can be determined by the needed lens movement to stay in the working range.

Usually optical sensors deliver up to nanometer resolution and allow a far higher measurement speed than tactile probes. Disadvantages are their lower lateral resolution compared to the working direction and dependence of surface characteristics like degree of reflectivity. Additionally, optical systems only allow $21 / 2 D$ measurements and are restricted to surface areas with low inclination. Another advantage of tactile measurement systems is their well known and standardized traceability procedures, which is not always the case in most optical systems.

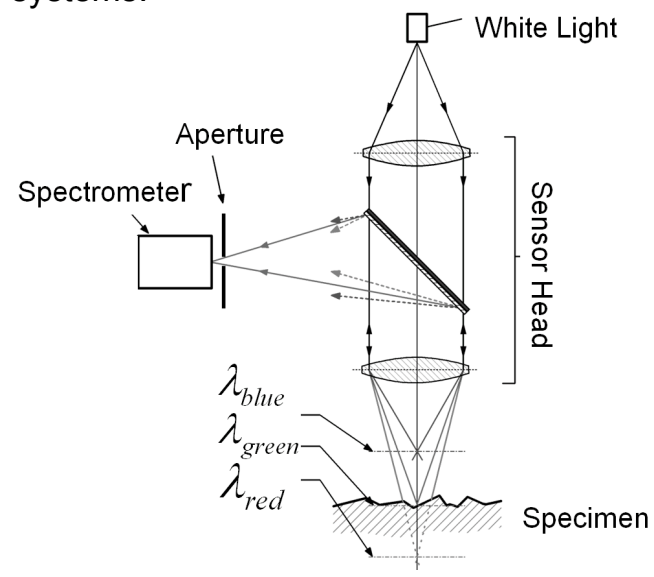

figure 6: chromatic sensor

\subsection{Scanning probe systems}

Additional to tactile and optical systems, there also exist sensors which work with scanning probe systems, like atomic force microscopes and scanning tunnelling microscopes. For their application in CMMs, the vibration amplitudes of the CMMs should be close to sub-nanometer otherwise the working range of the sensors would be exceeded. Therefore only metrological scanning probe microscopes or a few nano coordinate measurement machines like the NMM-1 can be used for theses probes [18]. Compared to other sensors, the scanning probes require a high surface quality of the working piece with characteristics like flatness, solidness or conductivity, depending on the used technique.

These preconditions get clear by looking at the working principle of e.g. the AFM, see figure 7 . To overcome the restrictions by the size of a laserspot, a cantilever with a sharp tip and a tip radius of only a few nanometer is used. The cantilever reflects a laserbeam onto a photosensitive device which detects the movement of the cantilever. The resolution of this system easily reaches nanometer or even sub nanometer.
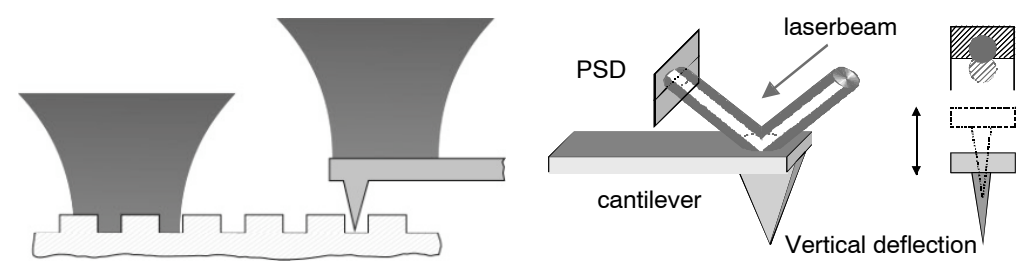

figure 7: Illustration of an AFM

An other scanning probe principle is used in scanning tunneling microscopes, which use the quantum physical phenomen of electron tunneling. A distance-dependent current between an atomic sharp tip and 
the surface atoms of the workpiece can be used for length measurement. This kind of working principle delivers atomic resolution, restricted by the tip quality, and was also implemented into the nano coordinate measuring machine NMM-1. Additionally by accepting limitations in the lateral resolution it was possible to use conductive spheres and thus creating a robust and isotropic probing system overcoming the fragility of scanning probe microscopes. [19], [20].

\section{CONCLUSIONS}

Miniaturized CMMs are appropriate for flexible measurements of form and dimensions of micro sized parts. In general they are set up out of the basic components: guiding and motion systems, metrology frame, probing system and software. While for length measuring systems only laser interferometers are used, there is a variety of different philosophies and solutions for all other mentioned devices. In this paper, not only the current scientific and technological approaches for most important components are demonstrated but also the most promising existent implementations are shown.

\section{REFERENCES}

[1] Christoph, R.; Neumann, H.J.: Multisensor-Koordinatenmesstechnik. München: sv corporate media, 2006; ISBN 3-937889-51-5.

[2] Jäger, G.; Manske, E.; Hausotte, T.; Büchner, H.-J.: Nanomessmaschine zur abbefehlerfreien Koordinatenmessung. In: tm - Technisches Messen 7-8 (2000), pp. 319-323.

[3] Ruij, T.: Ultra Precision Coordinate Measuring Machine. Eindhoven, 2001. ISBN 90-6464287-7.

[4] Schulz, D.: Rasterkraftmikroskope vom Typ Veritekt. PTB Braunschweig, 2005, http://www.ptb.de/de/org/5/52/525/rm/rm31_d.htm; as of 2009-02-04.

[5] PI Physikinstrumente: product overview, http://www.physikinstrumente.de/, as of 2009-02-04.

[6] Jäger, G.; Grünwald, R.; Manske, E.; Hausotte, T.; FüßI, R.: A Nanopositioning and Nanomeasuring Machine Operation - Measured Results. In: Nanotechnology and Precision Engineering, 6 (2004), Vol. 2, p. 81-84.

[7] Vermeulen, M.M.P.A.; Rosielle, P.C.J.N.; Schellekens, P.H.J.: Design of a high-precision 3Dcoordinate measuring machine. In: Annals of the CIRP 47/1/1998, pp. 447-450.

[8] Werth Messtechnik GmbH: product overview. http://www.werthmesstechnik.de/ as of 2009-02-04.

[9] Weckenmann, A.; Estler, T.; Peggs, G.; McMurtry, D.: Probing systems in dimensional metrology. In: Annals of CIRP, 53/2/2004, pp. 657-684.

[10] Weckenmann, A.; Peggs, G.; Hoffmann, J.: Probing systems for dimensional micro- and nanometrology. In: Measurement Science and Technology, 17 (2006), pp. 504-509.

[11] Peggs, G.N.; Lewis, A.J.; Oldfield, S.: Design for a compact high-accuracy CMM. In: Annals of CIRP 48/1/1999, pp. 417-420.

[12] Bütefisch, S.; Büttgenbach, S.: Taktiler Dreikomponenten-Kraftsensor. In: tm - Technisches Messen 66 (1999), pp. 185-190.

[13] Büttgenbach, S.; Brand, U., Bütefisch, S.; Herbst, Ch.; Krah, T., Phataralaoha; A., Tutsch, R.: Taktile Sensoren für die Mikromesstechnik: In: VDI Berichte 1950, Düsseldorf: VDI 2006, pp. 109118.

[14] Weckenmann, A.; Wiedenhöfer, T.; Büttgenbach, S.; Krah, T.; Fleischer, J.; Buchholz, I.; Viering, B.; Kranzmann, A.; Ritter, M.; Krüger-Sehm, R.; Bakucz, P.; Schmitt, R.; Koerfer, F.: Trends bei der Entwicklung von Normalen für die Mikro- und Nanomesstechnik - Herausforderungen und Lösungsansätze. In: tm - Technisches Messen 75 (2008), pp.288-297.

[15] Küng, A.; Meli, F.; Thalmann, R.: Ultraprecision micro-CMM using a low force 3D touch probe. In: Measurement Science and Technology, 18 (2007), pp. 319-327.

[16] Mitutoyo Vision \& Optical System Group, The UMAP Vision System, http://www.mitutoyo.co.jp/eng/solution/solution/electricity/pdf/umap.pdf as of 2009-02-04.

[17] Schwenke, H.; Waeldele, F., Weiskirch, C., Kunzmann, H., 2001, Opto-Tactile Sensor for 2D and 3D Measurement of Small Structures on Coordinate Measuring Machines. In: Annals of CIRP, 50/1/2001, pp. 361-364.

[18] Jäger, G.; Manske, E.; Hausotte, T. :Neue Anwendungen der Nanomessmaschine (NPMMaschine) durch die Entwicklung nanoskaliger optischer und taktiler Tastsensoren. In: tm - Technisches Messen 73 (2006), pp. 457-464.

[19] Weckenmann, A.; Hoffmann, J.: Long Range 3D Scanning Tunnelling Microscopy. In: Annals of CIRP 56/1/2007, pp. 525-528.

[20] Weckenmann, A.; Hoffmann, J.; Schuler, A.: Development of a tunnelling current sensor for a long-range nano-positioning device. : Measurement Science and Technology, 19 (2008), 064002, (7pp). 